\title{
Research on Various Electricity Price Forecasting Techniques for Indian Power Markets
}

\author{
Anamika
}

\begin{abstract}
A stable market Clearing rate (MCP) guaging machine is required for the two customers and electricity creators for succesful and worthwhile strength promote execution. This evaluation work predicts the marketplace Clearing fees (MCPs) for the extensive stretches of April, may and June using artificial Neural Networks (ANNs). the obvious charges and needs are accumulated from the Indian strength alternate (IEX) are used as records sources and centers for ANNs in looking ahead to MCPs. The hours with closeness in fees are gathered reliant on affiliation framework and peak-Off peak estimations of fees. Neural Networks are executed autonomously for each social affair to expect MCPs correctly. mean Absolute percentage mistakes (MAPEs) are evaluated to discover the great assembling machine and foreseeing version for Indian power Markets. MAPE effects are confirmed up within the first-class innovative days and the complete month for showing the ampleness of these get-together strategies. the connection amassing system is included because it indicates low MAPE for all instances. This examination offers an estimation to Indian power Markets as for the guaging model to screen and spoil down the instability in strength fees of IEX. this may assist the controllers with defining continuously effective processes on deregulation and modifications when compelling gauge opposition for the duration of strength markets modifications.
\end{abstract}

Keywords: -- Indian Electricity Markets, Artificial Neural Networks, Correlation grouping, Peak and Off Peak grouping

\section{INTRODUCTION}

Acquaintance of deregulation leads with patron driven, composed strength promote it, which offers open doors for ideal asset use and productive strength acquirement methodology. energy marketplace Clearing fee (MCP) is normally alluring cost of strength at concord wherein the market is clear of deficiency and overflow. For a unfastened framework administrator (ISO), MCP is acquired by explaining Unit dedication (UC) and financial Dispatch (ED) difficulty with the offers and framework situations. Henceforth, MCP determine is a key idea for marketplace individuals to augment their advantage. A first rate estimating model has fundamental importance of a properly operating commercial enterprise area and danger the board alternatives. strength pool and power trades are styles of looked after out marketplace, wherein day ahead strength is exchanged for following day or week day or for the duration of $60 \mathrm{mins}$, crest hours or off top hours. The marketplace Clearing prices (MCPs) in a deregulated strength market are volatile. The brilliant MCP expectation could aid utilities and autonomous power makers to put up compelling gives with low dangers and make incredible -sided exchanges.

many of the assortment of expectation techniques, neural structures have been broadly applied in mild of their solid nonlinear mapping and high level of precision. specific analysts have related ANNs to parent for temporary value estimating. the two figuring out strategies, to be unique

1. Forecasting established through and massive fees of similar days and,

2. Forecasting structured via and big expenses of similar days similarly to ANN refinement (ANN technique).

The parameters picked for ANN expectation depending on similar day's technique are: load at $\mathrm{t}$, load at $\mathrm{t}-1$, load at $\mathrm{t}+1$, cost at $\mathrm{t}$ and cost at $\mathrm{t}-1$. The precision of the anticipating strategy is progressed with the aid of the expansion of burden at $\mathrm{t}-1$ and $\mathrm{t}+1$. mean Absolute percent error (MAPE) and Root approach Squared error (RMSE) are picked as precision measures for the expecting fashions. The estimating fashions with determined facts resources are connected to PJM strength market to conjecture the LMPs for an stop of the week day in wintry weather, an occasion in spring and a overdue spring day. The quality MAPEs are discovered to be $5.33 \%, 5$.fifty three\% and $3.32 \%$ in my view [1].

The time association models Dynamic relapse (DR) and transfer work (TF) fashions are connected to gauge the genuine prices within the electricity markets of Mainland Spain and California. expenses from August 21 to 27, 2000 (low interest week) and November 13 to 19, 2000 (excessive hobby week) in the Spanish electricity market are determined. fees from April 3 to 9, 2000 in California energy markets are guage. The suggest of the errors is determined for all the three weeks and for both DR and TF models. each day imply errors are round $4.5 \%, 5 \%$ and $3 \%$ in the months August, November and April personally. Monday in the lengthy stretch of August is anticipated with excessive blunder. Hours with excessive charges are anticipated with excessive errors within the length of November. Spike costs are exactly anticipated within the long stretch of April [2]

To enlarge the precision of following day power value guaging the studying statistics for Neural Networks was first preprocessed via unpleasant set hypothesis. Connection outcomes demonstrate the connections in week after week variety statistics on electricity expenses. The \%MAPE for the year in PJM market is found to be 11-33\% [3]. 
Relative Volatility lists investigation is directed because of Ontario marketplace, where Volatility documents are created dependent on fee velocity ideas and verifiable unpredictability. The investigation is done in conditions.

1. Volatility statistics are resolved at the entire value time arrangement.

2. The price time arrangement of the day are separated into 24 squares and unpredictability information are determined for each unique square independently [4].

investigation of outcomes of presented institutional adjustments and administrative adjustments to the factors of strength costs in England and Whales cut price power exhibit at some stage in 1990 - 2001 are illustrated. value pinnacle guiding principle and Divestment arrangement are supplied by using administrative expert of notable of britain because of the improvement of value vacillation along interest. price top tenet done the goal of bringing down the cost degree at the fee of more costly charge instability [5].

Counterfeit Neural Networks are joined with portraits Processing units (GPUs) for expecting of intensity framework request in Korean energy Markets. As GPU is consolidated with ANN, MAPE of guaging end result is round $1 \%$ and figuring out end result is created short [6]. Non parametric relapse techniques are utilized for estimating the electricity value and request within the marketplace of terrain Spain [7].

Least square help Vector machine (LSSVM) is joined with car-Regressive transferring common with outer data (ARMAX) module to foresee hourly electricity MCP for an entire month. The LSSVM method is utilized as an essential approach to gauge the MCPs and ARMAX module is applied as a helper supporter to improve the watching for effects received from the preceding approach. The usage of $1 / 2$ breed procedure on PJM energy markets creates a MAE and MSRE of $2.7360 \%$ and zero. $1495 \%$ for my part. at the point whilst contrasted and the single LSSVM process, it's miles an improvement of one.eighty five $\%$ and $1.19 \%$ in MAE and RMSE for my part [8].

The LSSVM is being a solid relapse instrument to discern power cost in California's electricity markets. The information exams are collected, grouped and went thru LSSVM to expect value [9].

A Neural community based totally power MCP figuring out model is displayed on this paper to determine, hourly MCPs for an entire three months in advance. The 24 hours of multi day are assembled distinctively to acquire determining consequences close to the genuine estimations of MCP. accumulating of hours is accomplished dependent on techniques, especially: amassing depending on connection network and gathering dependent on top off top estimations of MCPs. MCPs of every amassing of hours are expected independently. The exhibition of the proposed estimating model is classified with the aid of verifiable data from Indian strength Markets [10].

Computational consequences affirmed that the proposed estimating model and amassing systems can enhance expectation exactness of MCPs contrasted with direct guaging of MCPs. The fundamental commitment of this paper incorporates

1. Modeling for reasonable watching for version for Indian energy Markets
2. Grouping of 24 hours of multi day dependent on courting framework

three. Grouping of 24 hours of multi day depending on top, off top estimations of MCPs.

The the rest of the paper is as consistent with the subsequent: section 2 outlines approachs utilized for estimating the MCPs. the gathering of hours depending on courting grid and crest, off pinnacle estimations of MCPs are depicted in segment 3. records resources and yields applied for anticipating the MCPs in Indian power Markets are talked about in area four. The discoveries of the exploration are introduced in phase 4 and ends are integrated into the area 5.

\section{APPROACHES}

\subsection{Artificial Neural Networks}

The interest in ANNs is essentially due to their skill to imitate herbal intelligence in its learning from experience. The architecture of ANN consists of many interconnected neurons with familiar traits consisting of enter, synaptic strengths, activation, output and bias. A Multi Layered Perceptron (MLP) Neural community (NN) using three hidden layers is applied on this studies for accurate fee forecasting. An MLP neural community may be regarded as three layered feed ahead neural community with a linear output mapping and with out comments. MLP-NN generally uses a linear transfer feature at output layer and nonlinear switch feature at hidden layers. The input layer absolutely consists of supply nodes connected by way of weighted connection to the hidden layer. allow's don't forget an synthetic neuron has $\mathrm{n}$ inputs as from the environment or adjacent neuron. Each input is weighted with before reaching the processing body of the neuron. The total signal net inside the neuron could be calculated by

$$
\text { net }=\sum_{i=1}^{n} x_{i} w_{i}=W^{T} X
$$

The weighted signal will be mapped by the activation function Hence the output can be calculated by

$$
y=f(\text { net })
$$

This yield may either contribution to the next neuron in nearby layer or last yield at the yield layer. The back proliferation calculation proposed by Paras Mandal et al [1] is utilized as a directed iterative preparing technique for discovering loads that can accomplish best mapping among info and yield. The loads update was done dependent on guideline expressed in (3). The iterative procedure of exhibiting an information yield pair and refreshing loads proceeds till the mistake capacity comes to a pre-indicated worth or loads never again change. All things considered the preparation stage is done and system is prepared for testing and activity. 


$$
w_{j i}^{n e w}=w_{j i}^{\text {old }}-\eta \frac{\partial E}{w_{j i}}
$$

where

$$
\text { the ith neuron and } j \text { th neuron }
$$

$\eta \quad=\quad$ learning rate valued between 0 and 1

And $E$ is the amount of error and it can be calculated as

$E=\sum_{\mathrm{k}=1}^{\mathrm{L}} \sum_{j=1}^{q}\left(b_{j k}-z_{k j}\right)^{2}$

where

$z_{j k}=\quad$ estimated value

The relationship offers the level of direct relationship among two factors, which entails how firmly the two factors are diagnosed with one another. it's miles finished by deciding the relationship coefficient, whose worth is limited between 1 and 1 . There are 3 capacity outcomes of being decidedly associated, contrarily linked and not corresponded for the relationship coefficients with features close to $1,-1$ and zero one at a time. The machine to sign up the relationship coefficients is the accompanying:

1. recall informational collections $\mathrm{X}$ and $\mathrm{Y}$ and convert them to conventional gadgets and determine imply and popular deviation of every datum set.

2. Compute the outcomes of the same old units of the $\mathrm{x}$-values and the $\mathrm{y}$-values.

three. Take the regular of the gadgets.

Connection is one of the most widely diagnosed and useful authentic exam instruments that can be applied in information displaying and research to evaluate degree of connection between factors. The point of this take a look at is to check which set of natural parameter may be very related to global sun Radiation. in this examination paintings, courting among's GSR and distinct different herbal variables are investigated and the parameters evaluating to better connection coefficient are applied to the faux neural gadget. the connection coefficient is gotten by means of using a nonparametric percentage of measurable reliance among two elements called Spearman rank-connection coefficient, whose one vast trademark is that it makes use of rank-request of the statistics paying little recognize to dispersion between informational collections. It depicts the diploma of courting between's two factors utilizing a monotonic capacity. it is affordable for discrete and consistent factors as well as ordinal factors. This makes it right for the informational indexes along with unordinary values as well, because the conflicting qualities have no mind-blowing effect on the assessed outcomes. The statement of Spearman rank-connection coefficient for its be counted is given as

$$
\rho=1-6 \sum_{i=1}^{n} D_{i}^{2} / n\left(n^{2}-1\right)
$$

$w_{j i}=$ weight between

$b_{j k}=$ actual target value

\subsection{Correlation Analysis}

Where,

$$
D_{i}=x_{i}-y_{i}
$$

\subsection{Performance Evaluation}

Mean absolute error (MAE), mean absolute percentage error (MAPE) and mean square root error (MSRE) are the three most widely used measurements for performance evaluation of forecasting MCPs. In this paper only MAPE is selected for regression computation performance evaluation. The expression to evaluate the MAPE is as follows.

$$
M A P E=\frac{1}{N} \sum_{t=1}^{N} \frac{\left|t_{k}-O_{k}\right|}{t_{k}} \times 100
$$

\section{GROUPING OF HOURS OF THE DAY}

This area offers the extraordinary gathering of hours for execution of estimating marketplace Clearing expenses (MCPs) for Indian power Markets. The 24 hours of the day may be partitioned into one-of-a-kind gatherings which make guaging mistake least. finally new methodologies are proposed which amassing the 24 hours of multi day based totally on similitude in fees. Out of the two methodologies the previous being the gathering dependent on courting community and later being the collection dependent on height and off height. the two strategies are as per the subsequent.

three.1.Grouping dependent on Correlation framework

relationship device is the most sizeable logical technique for selecting the quantity of gatherings of similar fee in multi day of 24 hours. extraordinary specialists applied courting investigation for dedication of contributions to an estimating version. in this exploration relationship examination is utilized to accumulate comparative hours dependent on MCPs. A connection grid has been assessed to find out the hours which can be almost corresponded. The lattice has been assessed depending on the records from first January, 2014 to 30th June, 2014 of Indian energy Markets. The grid became displayed within the desk 1 and uncovers that the 24 hours in multi day can be remoted into 5 gatherings and they are added as pursues.

gathering 1 carries the hours from 1 to five contingent on the relationship issue zero.77. table 1 uncovers that the hours $1,2,3,4$ are linked as zero.seventy nine, 0.88 , zero.ninety one and zero.ninety five personally, as for hour 5 which is extra than 0 .seventy seven.

accumulating 2 accommodates of hours 6, 7, eight and 9 that have the connection factors as zero.seventy eight, zero.79, zero.938 and 1 one after the other as for hour 9.

collecting 3 incorporates of hours 10 to 17 that have the connection thing as 0.87 , zero.87, zero.nine, 0.nine, 0.85 , $0.88,0.87$ and 1 individually regarding hour 17 . the connection aspect of hour 18 with 19 is zero.68 and 17 is zero.eighty three. This expects to embed the hour 18 with the gathering that incorporates hour 17.

collecting 4 besides the hour 19 isn't always related highly with any of the relaxation of the hours. alongside those lines, 
this hour is taken into consideration independently as a gathering.

gathering 5 establishes of hours 20, 21 and 22 that have the relationship component of $0.86,0$.ninety three and 1 one at a time. the connection elements of hour 23 with 22 and 24 are 0. nine and 0.eighty four individually. This prompts inclusion of super importance 23 into the gathering that carries hour 22.

\subsection{Grouping based on Peak and Off Peak values of MCP}

A normal subdivision in just double cross squares is proposed to figure the MCPs in the Indian Electricity Market. Table 2 speaks to the real dissemination of Peak and off Peak hours of consistently for thinking about the timeframe. Different blends of hours are discovered to find Peak and Off Peak hours dependent on the similitude on cost. Because of the restriction in ANNs (as preparing information ought to be like trying information), among the numerous blends of Peak and off Peak hours the most proper mix has been chosen. The accompanying figures speak to Peak and Off Peak esteemed hours uncovered in the wake of dealing with from different blends. The figures 1-6 speak to Peak and Off Peak hours of the months January, February, March, April, May and June. Every single month is watched distinctly and Peak and Off Peak hours are chosen. These figures uncover that the pinnacle hours are 10,11, 12, 13, 14, 15, 16, 20, 21, 22, 23 and 24 respectively. The remaining hours $1,2,3,4,5,6,7,8$, 9, 17, 18 and 19 are considered as Off Peak hours.

Table 1 Correlation Matrix of MCPs for the considered period.

\begin{tabular}{|c|c|c|c|c|c|c|c|c|c|c|c|c|c|c|c|c|c|c|c|c|c|c|c|c|}
\hline & 1 & 2 & 3 & 4 & 5 & 6 & 7 & 8 & 9 & 10 & 11 & 12 & 13 & 14 & 15 & 16 & 17 & 18 & 19 & 20 & 21 & 22 & 23 & 24 \\
\hline 1 & 1 & & & & & & & & & & & & & & & & & & & & & & & \\
\hline 2 & 0.93 & 1 & & & & & & & & & & & & & & & & & & & & & & \\
\hline 3 & \begin{tabular}{|l|}
0.89 \\
\end{tabular} & \begin{tabular}{|l|}
0.97 \\
\end{tabular} & 1 & & & & & & & & & & & & & & & & & & & & & \\
\hline 4 & 0.86 & \begin{tabular}{|l|}
0.94 \\
\end{tabular} & 0.96 & 1 & & & & & & & & & & & & & & & & & & & & \\
\hline 5 & \begin{tabular}{|l}
0.79 \\
\end{tabular} & \begin{tabular}{|l|}
0.88 \\
\end{tabular} & 0.91 & 0.95 & 1 & & & & & & & & & & & & & & & & & & & \\
\hline 6 & \begin{tabular}{|l|l|}
0.77 \\
\end{tabular} & \begin{tabular}{|l|}
0.82 \\
\end{tabular} & 0.84 & 0.87 & $\begin{array}{ll}0.9 \\
\end{array}$ & 1 & & & & & & & & & & & & & & & & & & \\
\hline 7 & 0.51 & \begin{tabular}{|l|}
0.59 \\
\end{tabular} & 0.65 & 0.68 & 0.74 & 0.79 & 1 & & & & & & & & & & & & & & & & & \\
\hline 8 & 0.66 & \begin{tabular}{|l|}
0.68 \\
\end{tabular} & 0.73 & 0.78 & 0.79 & 0.79 & 0.86 & 1 & & & & & & & & & & & & & & & & \\
\hline 9 & 0.65 & 0.66 & 0.71 & 0.74 & 0.75 & 0.78 & \begin{tabular}{|l|}
0.79 \\
\end{tabular} & 0.938 & 1 & & & & & & & & & & & & & & & \\
\hline 10 & 0.73 & 0.65 & 0.66 & 0.68 & 0.66 & 0.64 & 0.52 & 0.768 & 0.83 & 1 & & & & & & & & & & & & & & \\
\hline 11 & \begin{tabular}{|l|l|}
0.69 \\
\end{tabular} & \begin{tabular}{|l|}
0.57 \\
\end{tabular} & 0.58 & $\begin{array}{l}0.58 \\
\end{array}$ & \begin{tabular}{|l|}
0.54 \\
\end{tabular} & 0.51 & \begin{tabular}{|l|}
0.31 \\
\end{tabular} & \begin{tabular}{|l|l|}
0.594 \\
\end{tabular} & \begin{tabular}{|l|}
0.65 \\
\end{tabular} & 0.9 & 1 & & & & & & & & & & & & & \\
\hline 12 & \begin{tabular}{|l|}
0.69 \\
\end{tabular} & \begin{tabular}{|l|}
0.57 \\
\end{tabular} & 0.58 & 0.58 & \begin{tabular}{|l|}
0.53 \\
\end{tabular} & \begin{tabular}{|l|}
0.56 \\
\end{tabular} & \begin{tabular}{|l|}
0.34 \\
\end{tabular} & 0.593 & \begin{tabular}{|l|}
0.64 \\
\end{tabular} & $\begin{array}{l}0.86 \\
\end{array}$ & 0.94 & 1 & & & & & & & & & & & & \\
\hline 13 & \begin{tabular}{|l}
0.69 \\
\end{tabular} & \begin{tabular}{|l|}
0.58 \\
\end{tabular} & 0.58 & 0.59 & 0.54 & 0.55 & \begin{tabular}{|l|}
0.33 \\
\end{tabular} & 0.58 & 0.62 & 0.84 & 0.92 & 0.97 & 1 & & & & & & & & & & & \\
\hline 14 & 0.66 & \begin{tabular}{|l|}
0.56 \\
\end{tabular} & 0.55 & 0.56 & $\begin{array}{ll}0.52 \\
\end{array}$ & 0.56 & \begin{tabular}{|l|}
0.32 \\
\end{tabular} & \begin{tabular}{|l|l}
0.557 \\
\end{tabular} & 0.6 & 0.79 & \begin{tabular}{l|l}
0.85 \\
\end{tabular} & 0.89 & 0.92 & 1 & & & & & & & & & & \\
\hline 15 & \begin{tabular}{|l|}
0.69 \\
\end{tabular} & 0.57 & 0.55 & 0.55 & \begin{tabular}{|l|}
0.49 \\
\end{tabular} & 0.55 & \begin{tabular}{|l|}
0.31 \\
\end{tabular} & \begin{tabular}{|l}
0.516 \\
\end{tabular} & 0.57 & 0.77 & 0.82 & 0.89 & 0.91 & 0.95 & 1 & & & & & & & & & \\
\hline 16 & 0.66 & 0.52 & 0.5 & 0.48 & \begin{tabular}{|l|}
0.39 \\
\end{tabular} & 0.44 & \begin{tabular}{|l|}
0.19 \\
\end{tabular} & \begin{tabular}{|l}
0.438 \\
\end{tabular} & \begin{tabular}{|l|}
0.5 \\
\end{tabular} & 0.74 & 0.81 & 0.89 & 0.9 & 0.9 & 0.96 & 1 & & & & & & & & \\
\hline 17 & 0.74 & 0.65 & 0.63 & 0.62 & 0.57 & 0.56 & 0.35 & \begin{tabular}{|l}
0.591 \\
\end{tabular} & 0.65 & 0.87 & 0.87 & 0.9 & 0.9 & 0.85 & \begin{tabular}{|l|}
0.88 \\
\end{tabular} & \begin{tabular}{|l|}
0.87 \\
\end{tabular} & 1 & & & & & & & \\
\hline 18 & \begin{tabular}{|l|}
0.63 \\
\end{tabular} & \begin{tabular}{|l|}
0.64 \\
\end{tabular} & 0.65 & \begin{tabular}{l|l}
0.69 \\
\end{tabular} & \begin{tabular}{|l|}
0.69 \\
\end{tabular} & \begin{tabular}{|l|}
0.63 \\
\end{tabular} & \begin{tabular}{|l|l|}
0.52 \\
\end{tabular} & \begin{tabular}{|l|l|}
0.676 \\
\end{tabular} & \begin{tabular}{|l|}
0.7 \\
\end{tabular} & $\begin{array}{ll}0.77 \\
\end{array}$ & 0.74 & 0.73 & 0.72 & 0.7 & \begin{tabular}{|l|}
0.65 \\
\end{tabular} & \begin{tabular}{|l|}
0.58 \\
\end{tabular} & \begin{tabular}{|l|}
0.83 \\
\end{tabular} & 1 & & & & & & \\
\hline 19 & 0.32 & \begin{tabular}{|l|}
0.47 \\
\end{tabular} & 0.51 & 0.58 & 0.67 & 0.56 & \begin{tabular}{|l|}
0.53 \\
\end{tabular} & \begin{tabular}{|l|l|}
0.537 \\
\end{tabular} & \begin{tabular}{|l|}
0.54 \\
\end{tabular} & 0.46 & 0.36 & 0.31 & 0.32 & 0.3 & \begin{tabular}{|l|}
0.22 \\
\end{tabular} & \begin{tabular}{|l|}
0.09 \\
\end{tabular} & \begin{tabular}{|l|}
0.35 \\
\end{tabular} & 0.68 & 1 & & & & & \\
\hline 20 & \begin{tabular}{|l|l|}
0.59 \\
\end{tabular} & \begin{tabular}{|l|}
0.59 \\
\end{tabular} & 0.62 & 0.65 & \begin{tabular}{|l|}
0.67 \\
\end{tabular} & 0.72 & \begin{tabular}{|l|}
0.64 \\
\end{tabular} & 0.67 & 0.7 & 0.66 & 0.58 & 0.64 & 0.66 & 0.69 & 0.66 & \begin{tabular}{|l|}
0.57 \\
\end{tabular} & \begin{tabular}{|l|}
0.61 \\
\end{tabular} & 0.66 & \begin{tabular}{|l|}
0.64 \\
\end{tabular} & 1 & & & & \\
\hline 21 & 0.66 & 0.6 & 0.6 & 0.61 & 0.61 & 0.69 & 0.57 & \begin{tabular}{|l}
0.634 \\
\end{tabular} & 0.67 & 0.7 & 0.64 & 0.71 & 0.71 & 0.74 & 0.75 & 0.69 & \begin{tabular}{|l|}
0.68 \\
\end{tabular} & 0.61 & \begin{tabular}{|l|l|} 
\\
\end{tabular} & 0.93 & 1 & & & \\
\hline 22 & 0.76 & \begin{tabular}{|l|}
0.69 \\
\end{tabular} & 0.69 & 0.68 & 0.66 & 0.7 & 0.57 & \begin{tabular}{|l}
0.702 \\
\end{tabular} & 0.72 & 0.78 & 0.71 & 0.74 & 0.74 & 0.77 & 0.77 & \begin{tabular}{|l|}
0.72 \\
\end{tabular} & 0.76 & 0.67 & 0.42 & 0.86 & 0.93 & 1 & & \\
\hline 23 & \begin{tabular}{|l|l|}
0.76 \\
\end{tabular} & \begin{tabular}{|l|}
0.67 \\
\end{tabular} & 0.63 & 0.61 & \begin{tabular}{|l|}
0.57 \\
\end{tabular} & 0.64 & 0.46 & \begin{tabular}{|l|l|}
0.591 \\
\end{tabular} & \begin{tabular}{|l|}
0.62 \\
\end{tabular} & 0.74 & 0.69 & 0.74 & 0.73 & 0.78 & \begin{tabular}{|l|}
0.81 \\
\end{tabular} & \begin{tabular}{|l|}
0.79 \\
\end{tabular} & $\begin{array}{l}0.8 \\
\end{array}$ & \begin{tabular}{|l|}
0.64 \\
\end{tabular} & \begin{tabular}{|l|l|}
0.24 \\
\end{tabular} & 0.72 & 0.84 & 0.9 & 1 & \\
\hline 24 & \begin{tabular}{|l|l}
0.77 \\
\end{tabular} & \begin{tabular}{|l|}
0.75 \\
\end{tabular} & 0.71 & 0.7 & 0.65 & 0.64 & 0.5 & \begin{tabular}{|l|l}
0.606 \\
\end{tabular} & 0.62 & 0.7 & 0.63 & 0.65 & 0.65 & 0.63 & 0.66 & 0.63 & \begin{tabular}{|l|}
0.77 \\
\end{tabular} & 0.74 & 0.39 & 0.65 & 0.7 & 0.78 & 0.84 & \\
\hline
\end{tabular}

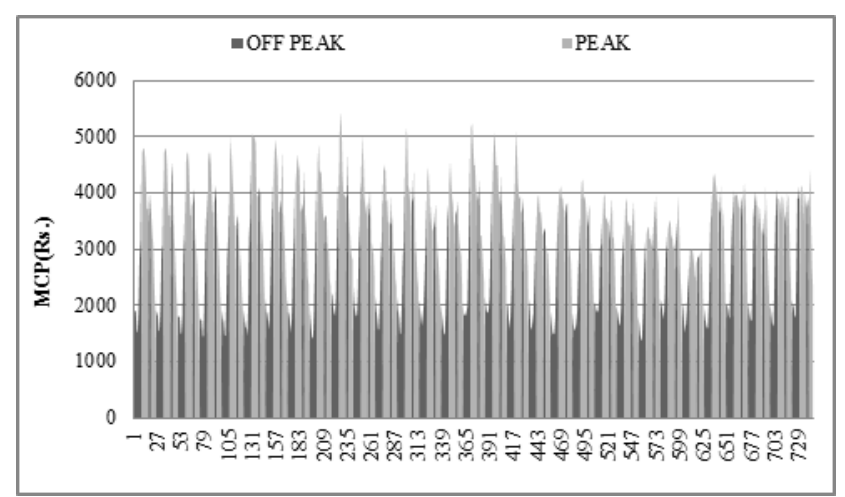

Fig. 1 Peak and Off Peak hours for the month of January

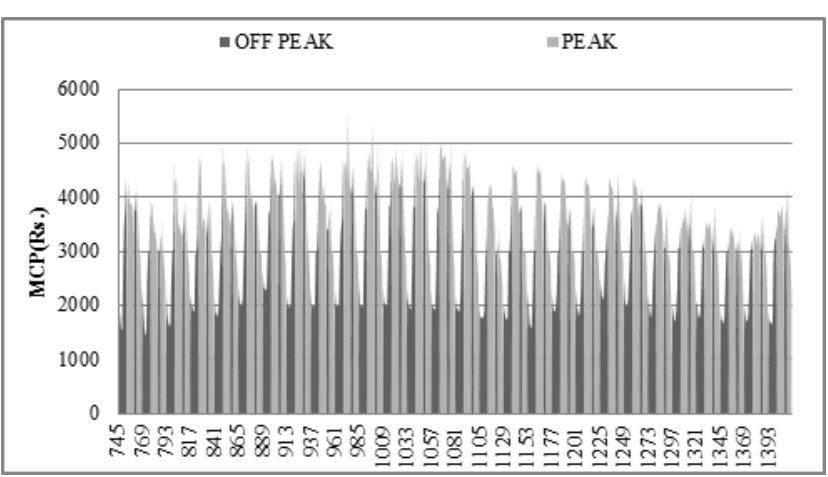

Fig. 2 Peak and Off Peak hours for the month of February 


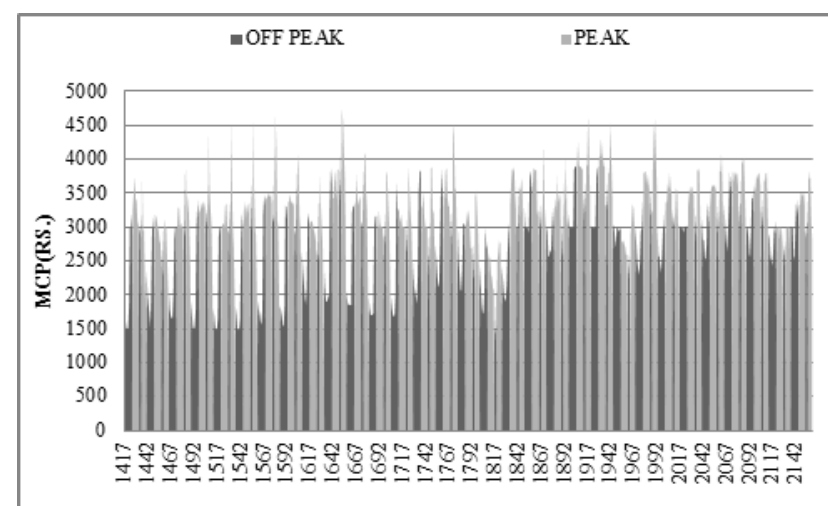

Fig. 3 Peak and Off Peak hours for the month of March

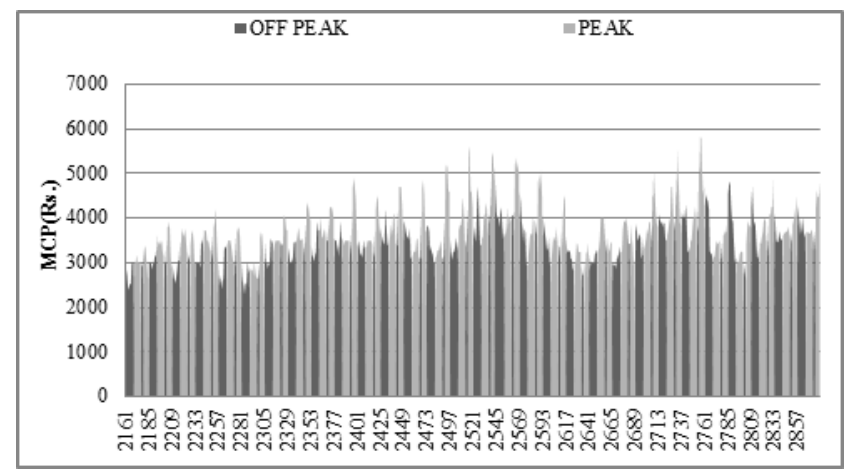

Fig. 4 Peak and Off Peak hours for the month of April

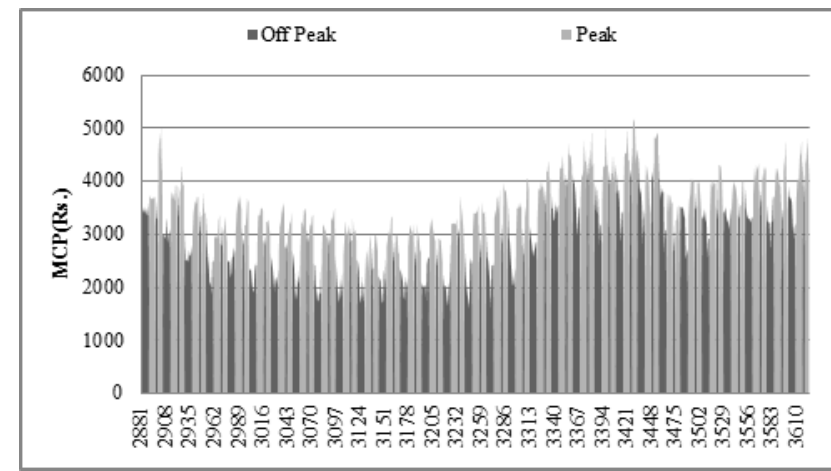

Fig. 5 Peak and Off Peak hours for the month of May

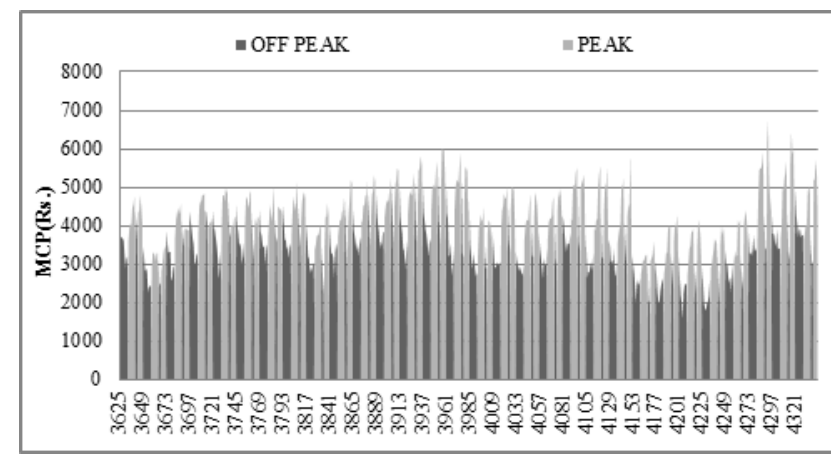

Table 2 Classification of hours for the Peak, Off Peak hours grouping technique

\begin{tabular}{|l|l|l|l|}
\hline Data set & Month & Peak Hours & Off Peak Hours \\
\hline \multirow{4}{*}{ Training } & January & $9,10,11,12,13$ & $\begin{array}{l}1,2,3,4,5,6,7, \\
8,14,15,16,17, \\
18,19,20,21, \\
22,23,24\end{array}$ \\
\cline { 2 - 4 } & February & $9,10,11,12,13$ & $\begin{array}{l}1,2,3,4,5,6,7, \\
8,14,15,16,17,\end{array}$ \\
\hline
\end{tabular}

Fig. 6 Peak and Off Peak hours for the month of June

\begin{tabular}{|c|c|c|c|}
\hline & & & $\begin{array}{l}18,19,20,21, \\
22,23,24\end{array}$ \\
\hline & March & $\begin{array}{l}10,11,12,13, \\
14,19,20,21,22\end{array}$ & $\begin{array}{l}1,2,3,4,5,6,7 \\
8,9,15,16,17 \\
18,23,24\end{array}$ \\
\hline \multirow{3}{*}{ Testing } & April & $\begin{array}{l}10,11,12,13, \\
14,19,20,21,22\end{array}$ & $\begin{array}{l}1,2,3,4,5,6,7 \\
8,9,15,16,17 \\
18,23,24\end{array}$ \\
\hline & May & $\begin{array}{l}10,11,12,13, \\
14,15,16,20, \\
21,22,23,24\end{array}$ & $\begin{array}{l}1,2,3,4,5,6,7 \\
8,9,17,18,19\end{array}$ \\
\hline & June & $\begin{array}{l}11,12,13,14, \\
15,16,20,21, \\
22,23,24\end{array}$ & $\begin{array}{l}1,2,3,4,5,6,7 \\
8,9,10,17,18 \\
19\end{array}$ \\
\hline
\end{tabular}

\section{INPUTS AND OUTPUTS RESULTS}

The facts assets and yields are selected for figuring out MCP in Indian electricity Markets dependent on information given by using Deepak Singhal et. al. [11]. Time steps are hourly, which mean that one day includes 24 hours. The facts are accrued from the Indian electricity alternate (IEX) [10] for a half yr from January 2014 to June 2014. The neural systems are prepared with half of statistics and tried with residual 50 $\%$ information.

Verifiable records on strength prices and beyond burden request establishes widespread contributions for foreseeing MCP. The different records assets considered for looking ahead to the MCPs are tested as pursues.

- Day of the week is the parameter as it recognizes the week via week off day and week days.

- A time table emptiness of Day is a sizable parameter that is useful in distinguishing the top and rancid top hours.

- Guage call for is the parameter that characterizes the interest at every hour.

- exchange in call for is characterized because the distinction among the requests of gift and in advance hours.

- value sooner or later lower back is the value at the sooner day identical hour. 3 back to returned time ventures for cost one day previous are considered as resources of data.

- fee multi week earlier is characterised as the cost earlier than seven days of that day and equal hour. 3 successive time ventures for fee multi week again are considered as information resources.

- price two weeks previous is characterized as the cost before approximately fourteen days of that day and identical hour.

- value three weeks earlier is characterised as the price earlier than three weeks of that day and identical hour.

- Fee a month lower back is characterised as the fee earlier than approximately a month of that day and equal hour.

- five. outcomes

The electricity value and request information had been accumulated for a half 12 months from IEX [10]. $50 \%$ information are applied for getting ready and the rest of the 50 $\%$ is applied for trying out neural systems. The time steps are hourly, which means that every day contains of 24 levels

\section{Published By:}


(i.e., 24 hours). The proposed version would require enormous investment documents, verifiable expenses and chronicled request as records sources and foresee marketplace Clearing charge. Neural Networks are connected independently on diverse gatherings to figure hourly MCPs for the long intervals of April, may and June of the yr 2014 in the Indian energy alternate.

\subsection{Correlation Grouping outcomes}

the connection division of gatherings is applied to figure electricity cost of similar hours in a second which grants low guaging blunder. the collection vicinity gives numerous gatherings into which 24 hours of the day are assembled. in the beginning, these are partitioned depending on relationship lattice. sooner or later five separate artificial Neural Networks are applied to figure MCPs for the lengthy stretches of April, might also and June individually. The feed ahead structures had been prepared to assess MCP depending on the training informational collection. The great version is approved via examination amongst evaluated and anticipated estimations of MCPs.

The figure $7 \mathrm{a}$ portrays the genuine and evaluated estimations of marketplace Clearing fee for the Indian energy trade inside the lengthy stretch of April (for just back to back days). The diagram demonstrates a nearly understanding among assessed and actual estimations of MCP. The discern likewise uncovers that maximum intense MCP is on the market at 21st hour and least is at 28th hour. The relapse plot between the assessed estimation of MCP and the real estimation of MCP for the long stretch of April is seemed in fig. $7 \mathrm{~b}$ with the relapse coefficient (R2) of zero.9175. This ensures the closeness of the assessed an incentive with the genuine really worth. each the figures $7 \mathrm{a}$ and $7 \mathrm{~b}$ discover that evaluated estimations of MCPs pursue a similar sample as the real MCPs aside from at the 39th and 45th hours.

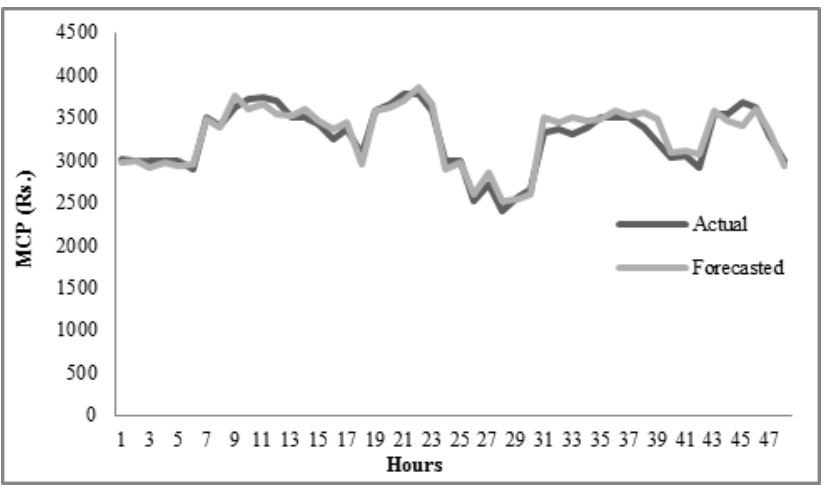

Fig. 7a Forecasted MCP and Actual MCP for two consecutive days in month April using correlation grouping

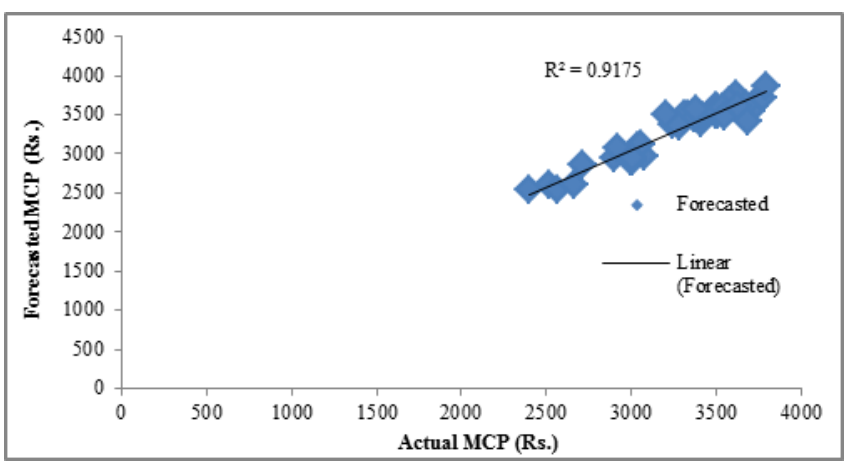

Fig. 7b Scatter plot between MCP and Actual MCP for two consecutive days in month April using correlation grouping

The decided and actual estimations of MCPs for IEX in the long stretch of might also are added inside the determine $8 \mathrm{a}$. The determine demonstrates a worth information amongst assessed and genuine estimations of MCP for all the 48 hours. The restriction of MCP is visible at twenty first hour and least is seen at 26th hour. discern $8 \mathrm{~b}$ demonstrates a relapse plot among evaluated and authentic estimations of MCPs for the lengthy stretch of may with R2 at 0.9438 . This determines a worthy information amongst expected and actual estimations of MCPs. The hours ninth and eighteenth are anticipated with less exactness within the long stretch of may additionally.

determine 9a portrays predicted and genuine estimations of MCPs for the long stretch of June. The most intense and least estimations of MCPs are visible at the forty seventh hour and the twenty ninth hour individually. dissipate plot among actual and ANN registered MCP in the lengthy stretch of June for considering two returned to again days are brought in figure $9 \mathrm{~b}$. severa deviations of records focuses from 1:1 line are visible in this month. The estimation of the coefficient of relapse is seen at zero.9192. the very best estimation of overall mistake within the lengthy stretch of June is visible at 31 st hour.

five.1. top and stale top Grouping effects

the peak and stale top lengthy stretches of collection is attached to estimate charges of height hours and rancid top hours of multi day. This sort of collection partitions absolutely the 24 hours into double cross squares and these are predicted independently. sooner or later synthetic Neural Networks are applied to determine MCPs for the lengthy stretches of April, may additionally and June respectively.determine $10 \mathrm{a}$ depicts the actual and assessed estimations of market Clearing price for the IEX within the length of April (for just two lower back to returned days) utilising top, off height amassing. The Graph demonstrates an ok knowledge amongst evaluated and real estimations of MCPs. The parent likewise uncovers that most severe MCP is accessible at 46th hour and least is at twenty eighth hour. The relapse plot between the assessed and the actual estimation of MCP for the lengthy stretch of April is appeared in fig. 10b. The coefficient of relapse (R2) is visible at zero.7279. This uncovers the surmised closeness of the assessed an incentive 
with the real well worth. both the figures $10 \mathrm{a}$ and $10 \mathrm{~b}$ uncover that MCPs at 29th hour and 22nd are anticipated with most elevated errors. the mistake gotten by peak, off height amassing at forty fifth hour is low while contrasted and mistake obtained by way of connection collecting.

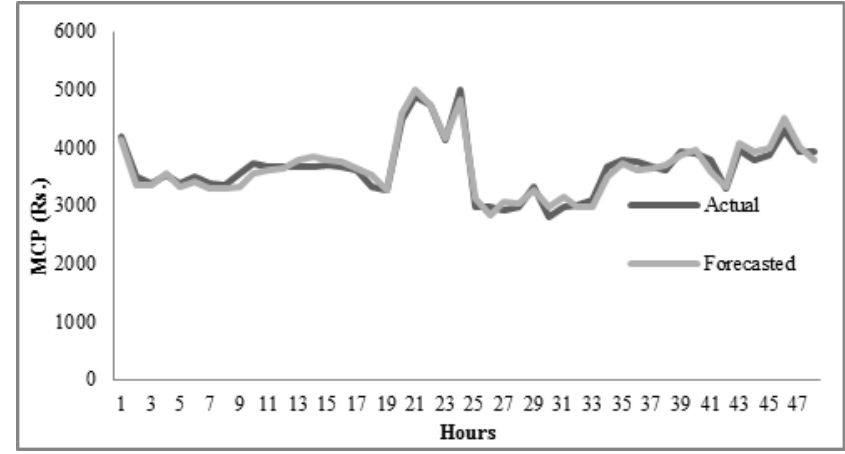

Fig. 8a Forecasted MCP and Actual MCP for two consecutive days in the month May using correlation grouping

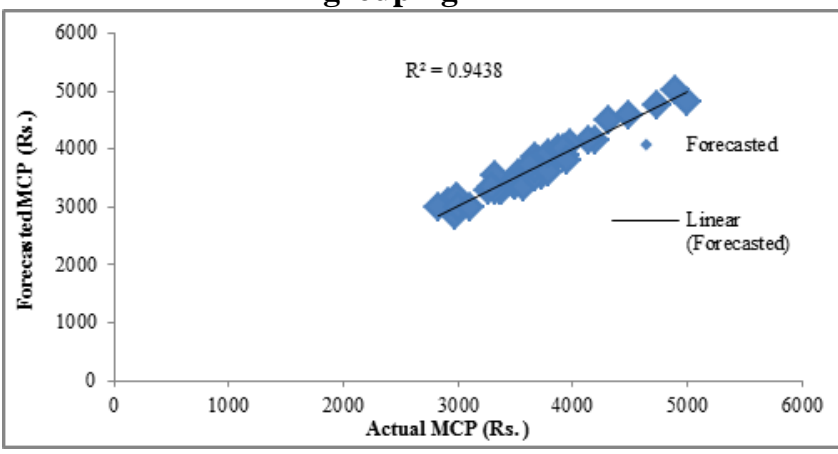

Fig. 8b Scatter plots for the Forecasted MCP and Actual MCP for two consecutive days in the month May using correlation grouping

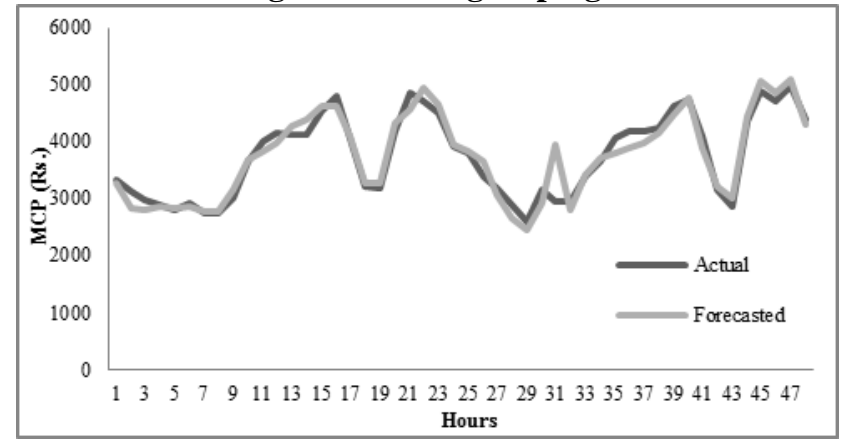

Fig. 9a Forecasted MCP and Actual MCP for two consecutive days in month June using correlation grouping

The forecasted and actual values of MCPs for IEX inside the month of may additionally are presented in determine $11 \mathrm{a}$. The figure shows a closest settlement among expected and real values of MCP for all the forty eight hours. The maximum of MCP is discovered at 21st hour and minimum is discovered at thirty third hour. discern $11 \mathrm{~b}$ suggests the regression plot between the envisioned price of MCP and the real cost of MCP for the month may also. The coefficient of regression is observed at 0.9127. This illustrates an excellent agreement among forecasted and real values of MCP. The hours except 8 th and 9 th are forecasted with appropriate accuracy.

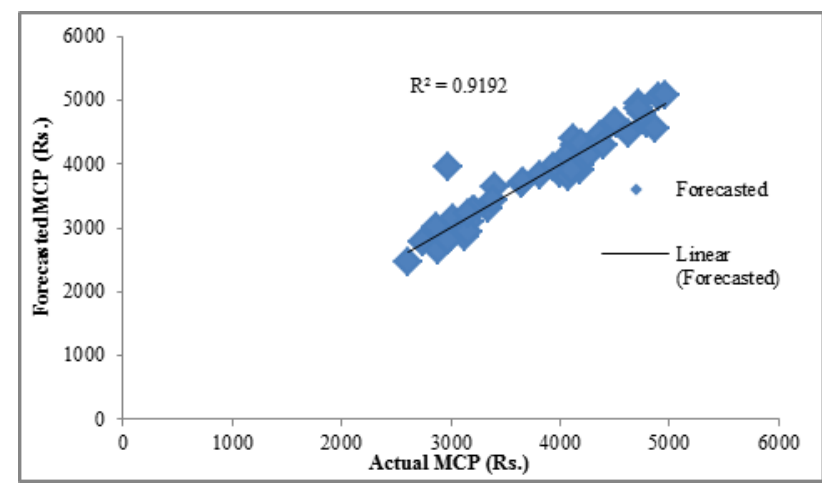

Fig. 9b Scatter plot for Forecasted MCP and Actual MCP for two consecutive days in month June using correlation grouping

The figure 12a delineates the anticipated and genuine estimations of MCP for the long stretch of June. The most extreme and least estimations of MCP are seen at sixteenth hour and the 29th hour separately. Dissipate plot among real and ANN processed MCP in the long stretch of June for considering two sequential days are displayed in figure $12 \mathrm{~b}$. Numerous deviations of information focuses from 1:1 line are seen in this month. The estimation of the coefficient of relapse is seen at 0.8934 . The most astounding estimation of total blunder in the period of June is seen at fifteenth hour.

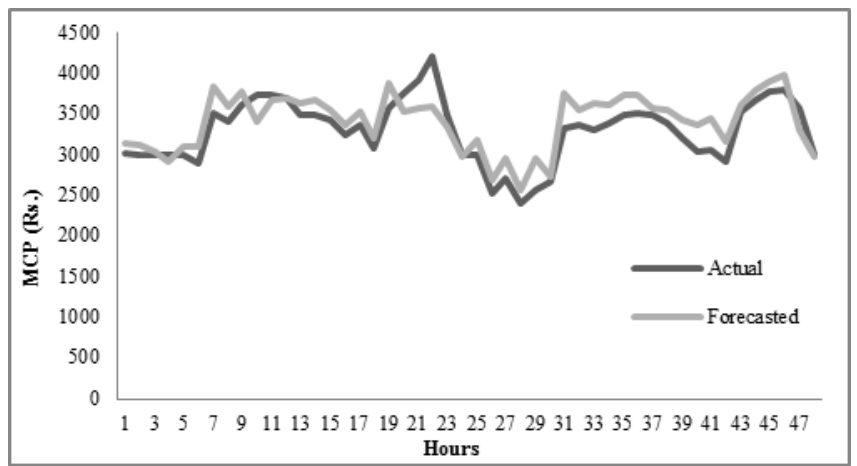

Fig. 10a Forecasted MCP and Actual MCP for two consecutive days in month April using Peak, Off Peak grouping

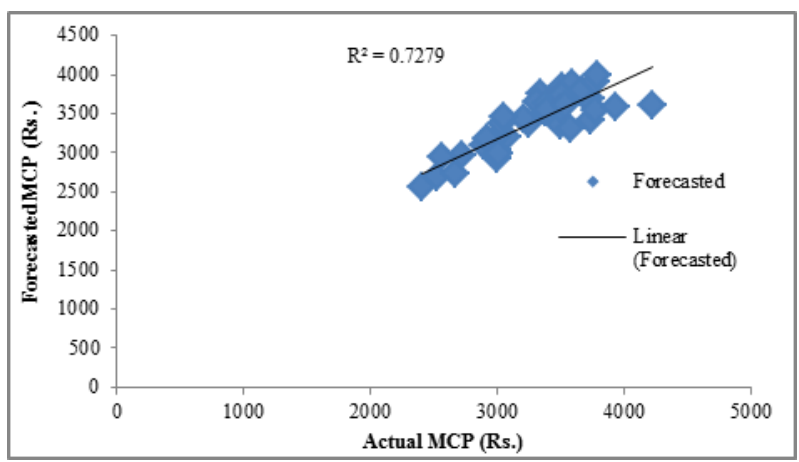

Fig. 10b Scatter plots for the forecasted MCP and actual MCP for two consecutive days in month April using Peak, Off Peak grouping

Published By: 


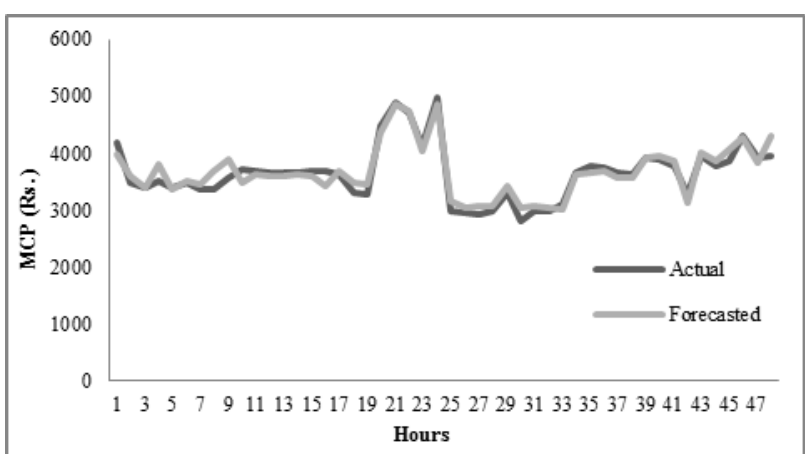

Fig. 11a Forecasted MCP and Actual MCP for two consecutive days in the month May using Peak, Off Peak grouping

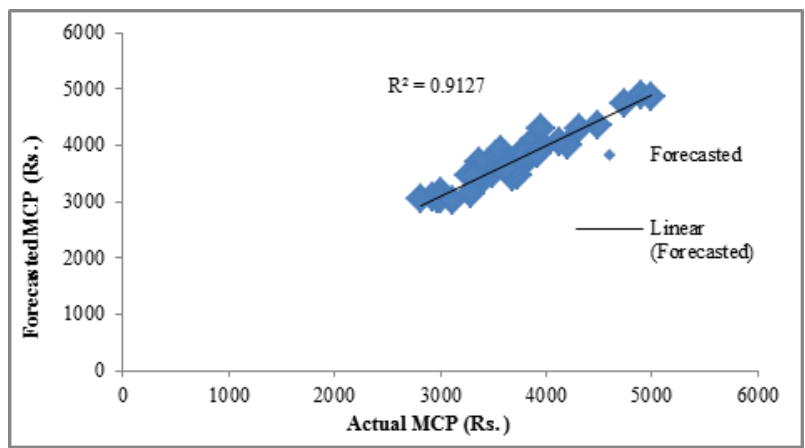

Fig. 11b Scatter plot for forecasted MCP and actual MCP for two consecutive days in the month May using Peak, Off Peak grouping

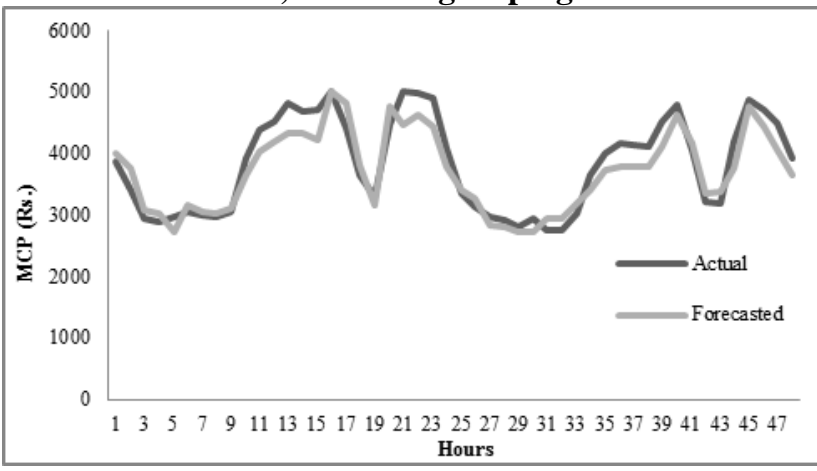

Fig. 12a Forecasted MCP and Actual MCP for two consecutive days in month June using Peak, Off Peak grouping

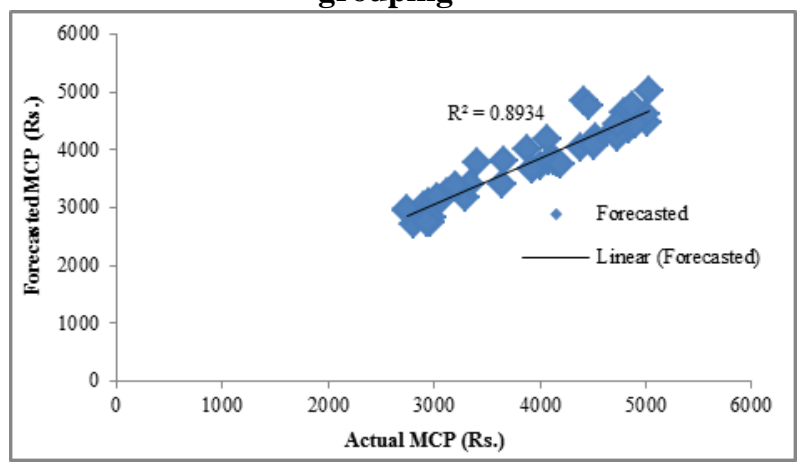

Fig. 12b Scatter plot for the forecasted MCP and actual MCP for two consecutive days in month June using Peak, Off Peak grouping

The MAPE esteems are moreover assessed for further choice within the investigation of precision. The estimations of outright errors, as indicated through the circumstance (5) for 2 sequential days and for the entire month using each connection and top, off peak gathering strategies are assessed. The MAPE esteems for three months by way of both of collection processes are organized in desk three. This affirms tremendous possibility in foreseeing marketplace Clearing rate by means of ANNs for Indian electricity markets. The MAPE esteems for 2 back to again days and entire month uncovers that connection amassing of hours creates much less blunder and very outstanding at expectation.

Table 3 MAPE results in two consecutive days and for whole month using both of the grouping techniques

\begin{tabular}{|c|c|c|c|c|}
\hline \multirow[b]{2}{*}{$\begin{array}{c}\text { Mont } \\
\mathrm{h}\end{array}$} & \multicolumn{2}{|c|}{ Correlation Grouping } & \multicolumn{2}{|c|}{ Peak, Off Peak grouping } \\
\hline & $\begin{array}{c}\text { Two } \\
\text { consecutive } \\
\text { days } \\
\end{array}$ & $\begin{array}{l}\text { Whole } \\
\text { Month }\end{array}$ & $\begin{array}{c}\text { Two } \\
\text { consecutive } \\
\text { days } \\
\end{array}$ & Whole Month \\
\hline April & $2.602 \%$ & $\begin{array}{c}09.9786 \\
\%\end{array}$ & $5.8838 \%$ & $09.1938 \%$ \\
\hline May & $2.776 \%$ & $\begin{array}{c}14.2805 \\
\%\end{array}$ & $3.2127 \%$ & $32.0518 \%$ \\
\hline June & $4.142 \%$ & $\begin{array}{c}14.0128 \\
\%\end{array}$ & $6.1237 \%$ & $24.8935 \%$ \\
\hline
\end{tabular}

\section{CONCLUSIONS}

in this paper, power marketplace Clearing fees estimation using Neural Networks is displayed in deregulated strength markets. The MCPs are assessed throughout the lengthy periods of April, may additionally and June for Indian energy alternate utilising a innovative gadget. unique gathering modules are linked at the start on accrued data to bring together the 24 hours of multi day. the connection collecting module partitioned the 24 hours of multi day into five gatherings. the height, off height accumulating module isolated the 24 hours of multi day into 2 gatherings. time table vacancies, proper requests and charges are used as contributions to the estimating apparatus. The MLP machine has been connected to conjecture the MCPs for Indian strength trade. The approval of the proposed model turned into finished with obscure facts, which the version did not see previously. exam of MAPE traits motives that the expectancy thru the proposed MLP system is increasingly appropriate and precise when contrasted with the alternative observational models. further, the relapse exam is moreover exhibited to defeat squaring organized unbalanced load in RMSE outcomes. The exam research on various amassing modules demonstrates that relationship primarily based collecting of hours will foresee the MCPs at leading precision over a few different systems. typically the proposed incorporated system has demonstrated an advanced waiting for precision contrasted with fundamental guaging techniques while related to numerous power markets.

\section{REFERENCES}

1. Paras Mandal, Tomonobu Senjyu, Atsushi Yona, Jung-Wook Park and Anurag okay. Srivastav, "Affectability analysis of similar Days Parameters for Predicting short time period energy rate", in Proc. Rests 07, 2007, pp. 568-574.

Published By: Blue Eyes Intelligence Engineering \& Sciences Publication 
2. Francisco J. Nogales, Javier Contreras, Antonio J. Conejo and Rosario Espínola, "Guaging subsequent Day electricity costs via Time series models", IEEE Trans. electricity systems, vol. 17, no. 2, pp. 342-348, may 2002.

3. Hirofumi Toyama, Tomonobu Senjyu, Phatchakorn Areekul, Shantanu Chakraborty, Atsushi Yona and Toshihisa Funabashi, "Following Day energy charge Forecasting on Deregulated power market" in Proc. T\&D Asia 2009, pp. 1-4.

4. 4.Hamidreza Zareipoura, Kankar Bhattacharya and Claudio A. Can izares, "electricity market price Volatility: the instance of Ontario", power policy, vol. 35, pp. 4739-4748, may additionally 2007.

5. Sherzod N. Tashpulatov, "Assessing the Volatility of strength costs: the instance of britain and Wales cut price strength showcase", energy policy, vol. 60, pp. 81-ninety, may additionally 2013 .

6. 6.Ting He, Ke Meng, Zhao Yang Dong, Yong-Taek Oh and Yan Xu, "utilization of excessive overall performance portraits Processing gadgets for energy gadget call for Forecasting", journal of electrical Engineering and generation, Vol. 5, No. three, pp. 363-370, may additionally 2010 .

7. 7.Juan M. Vilar, Ricardo Cao and Germán Aneiros, "expecting following day energy request and cost making use of non parametric utilitarian techniques," electric strength and strength structures, vol. 39, pp. 48-fifty five, Feb. 2012

8. 8.Xing Yan and Nurul A. Chowdhury, "Mid-term strength market clearing cost guaging: A half breed LSSVM and ARMAX method," electric power and strength structures, vol. fifty three, pp. 20-26, April 2013.

9. 9.Li Xie and Hua Zeng, "energy charge Forecasting by way of Clustering-Least Squares assist Vector device," in Proc. CSIP 2013, pp. 1357-1361.

10. 10. costs at IEX INR/Mwh, http://www.iexindia.com/marketdata/areaprice.aspx. Date final got to: 09.01.2015.

11. 11. Deepak Singhal and ok. S. Swarup, "electricity rate Forecasting making use of artificial Neural Networks", electric power and strength systems, vol. 33, pp. 550-555, Feb. 2011. 